\title{
EDUCAÇÃO EM SAÚDE COM CUIDADORES INFORMAIS DE PESSOAS IDOSAS COM DOENÇA DE ALZHEIMER: PESQUISA-AÇÃO
}

\section{HEALTH EDUCATION WITH INFORMAL CAREGIVERS OF ELDERLY PEOPLE WITH ALZHEIMER'S DISEASE: ACTION RESEARCH}

\section{Thaynara Maria Oliveira de Albuquerque ${ }^{1} *$ Daniele Pereira Soares $^{2} *$ Isabela Lunara Alves $^{*}$ Barbalho $^{3} *$ Marcelo Costa Fernandes ${ }^{4}$}

\section{RESUMO}

Objetivo: descrever a educação em saúde com cuidadores informais de pessoas idosas com doença de Alzheimer. Método: trata-se de estudo descritivo com abordagem qualitativa, embasado na pesquisa-ação, realizado com 15 cuidadores informais do município de São José de Piranhas, Paraíba, Brasil. Para a coleta de dados foi utilizada entrevista semiestruturada, após a aprovação do Comitê de Ética e Pesquisa. Utilizou-se do Discurso do Sujeito Coletivo para organização e análise dos resultados. Resultados: Observou-se que as metodologias ativas utilizadas proporcionaram espaço de troca de conhecimentos, além de modificar a percepção do cuidador sobre a Doença de Alzheimer. Considerações finais: é necessário promover a educação aos cuidadores buscando sanar as principais dificuldades em relação ao cuidado, proporcionando a ressignificação do cuidado a pessoa idosa com Doença de Alzheimer.

Palavras-chave: Cuidadores; Doença de Alzheimer; Educação em Saúde.

\begin{abstract}
Objective: to describe health education with informal caregivers of elderly people with Alzheimer's disease. Method: descriptive study, with a qualitative approach, based in the action-research, carried out with 15 informal caregivers in the municipality of São José de Piranhas, Paraíba, Brazil. In order to collect data, we used a semi-structured interview, after approval by the Research Ethics Committee. The Discourse of the Collective Subject was used to organize and analyze the results. Results: It was observed that the active methodologies used provided a space for exchanging knowledge, in addition to modifying the caregiver's perception of Alzheimer's Disease. Final considerations: it is necessary to promote education for caregivers, seeking to remedy the main difficulties in relation to care, providing a redefinition of care for the elderly person with Alzheimer's Disease
\end{abstract}

Keywords: Caregivers; Alzheimer Disease; Health Education.

\footnotetext{
${ }^{1}$ Enfermeira. Universidade Federal de Campina Grande. Cajazeiras, Paraíba, Brasil. ORCID: https://orcid.org/0000-0003-3804-3842

${ }^{2}$ Enfermeira. Residente do Programa de Residência Multiprofissional em Saúde da Família e Comunidade. Faculdade de Ciências Médicas da Paraíba/Secretaria Municipal de Saúde. João Pessoa, Paraíba, Brasil. ORCID: https://orcid.org/0000-0001-8575-5880.

${ }^{3}$ Graduanda em Enfermagem. Universidade Federal de Campina Grande. Membro da linha de pesquisa de Saúde Coletiva do Laboratório de Tecnologias de Informação e Comunicação em Saúde - LATICS. Cajazeiras, Paraíba, Brasil. ORCID: https://orcid.org/0000-0001-5672-4655.

${ }^{4}$ Enfermeiro. Doutor em Cuidados Clínicos em Enfermagem e Saúde pela Universidade Estadual do Ceará. Docente da Universidade Federal de Campina Grande. Líder do Grupo de Pesquisa Laboratório de Tecnologias de Informação e Comunicação em Saúde LATICS. Cajazeiras, Paraíba, Brasil. E- ORCID: https://orcid.org/0000-0003-1626-3043.
} 


\section{INTRODUÇÃO}

A Doença de Alzheimer (DA), devido suas repercussões na saúde, representa grande problema na atual sociedade, visto que compromete de modo profundo a integridade física, mental e social do sujeito acometido, exigindo cuidados mais complexos e gerando mudanças no cotidiano familiar ${ }^{(1,2)}$.

A DA é uma doença cerebral, sendo a mais comum entre as demências, danificando e destruindo partes do cérebro que envolvem o pensamento, a aprendizagem e a memória. À medida que a doença progride, os neurônios em outras partes do cérebro são afetados, fazendo com que as funções corporais mais básicas sejam prejudicadas, impondo ao indivíduo cuidados integrais por outra pessoa $^{(3)}$.

A pessoa idosa passa a depender totalmente de um cuidador, esse papel frequentemente é desenvolvido por um familiar ou pessoa próxima ${ }^{(4)}$. Esse tipo de cuidado se denomina cuidado informal, visto que a prática é exercida por pessoas sem formação na área e sem remuneração ${ }^{(5)}$.

Tendo em vista a complexidade da condução do cuidado pelo cuidador informal, nota-se predisposição para sobrecarga física, psicológica, financeira e social, principalmente quando não há formação técnico-científica, podendo aumentar a vulnerabilidade devido à ausência de orientações $^{(6)}$.

Vale salientar que a participação do cuidador em grupos de capacitação e orientação pode favorecer a troca de experiências e possibilitar constante atualização $^{(7)}$.

Desta forma, as práticas de educação em saúde, são estratégias que devem ser implementadas no cotidiano dos cuidadores informais, para o compartilhamento de saberes e vivencias e disseminação do conhecimento científico atrelado ao conhecimento cultural, diminuindo as vulnerabilidades existentes.

Com isso, surgiu a seguinte questão norteadora: A educação em saúde é um meio de orientar os cuidadores informais de idosos com doença de Alzheimer?

Este estudo apresenta a relevância de contribuir na percepção do cuidado dos cuidadores informais, impactando de forma positiva seu entendimento sobre a doença e valorizando a troca de experiências dos cuidados ofertados a pessoa idosa com DA.

Logo, objetivou-se descrever a educação em saúde com cuidadores informais de pessoas idosas com doença de Alzheimer.

\section{MÉTODO}

A pesquisa em questão é um recorte de um Trabalho de Conclusão de Curso de 
graduação em enfermagem da Universidade Federal de Campina Grande (UFCG), campus Cajazeiras. O estudo possui natureza descritiva, com abordagem qualitativa, alicerçada no método da pesquisa-ação ${ }^{(8)}$. Foi realizado na cidade de São José de Piranhas, uma cidade de pequeno porte, com estimativa de 20.329 habitantes segundo o Instituto Brasileiro de Geografia e Estatística (IBGE) ${ }^{(9)}$ que está localizada no alto sertão do estado da Paraíba, Brasil.

Os participantes dessa pesquisa foram os cuidadores informais de idosos portadores da DA em todo território que abrange a cidade de São José de Piranhas, no estado da Paraíba. Foi utilizado como critério de inclusão: cuidadores de idosos que independente de formação acadêmica exerça cuidado direto e domiciliar a um familiar ou não, sendo o idoso portador de laudo comprobatório de Doença de Alzheimer. Como critério de exclusão: cuidadores que não se encontrarem nas residências após três visitas consecutivas para o levantamento dos problemas e situações vivenciadas.

Após a constatação das problemáticas levantadas na primeira etapa do diagnóstico situacional, foram colocadas em prática as ações por meio dos círculos de cultura, rodas de conversa, seminários e ateliê, a fim de valorizar a participação ativa dos cuidadores, tornando-os protagonistas na construção de alternativas para solucionar as questões que ainda envolvem a Doença de Alzheimer nesse segmento populacional.

As ações foram desenvolvidas no Auditório Central Maria Elza, sendo realizadas em dois encontros, cada encontro durou em média duas horas e trinta minutos, em que no primeiro e segundo encontro, fizeram-se presentes sete e oito cuidadores, respectivamente.

As ações foram realizadas nos meses de outubro e novembro de 2018, e posteriormente, ocorreram as coletas pósintervenções, no mês de novembro com as seguintes questões: $O$ que significou para você a experiência em participar dessas ações educativas? Que sugestões você poderia acrescentar para a realização de novos grupos educativos? Após as ações educativas entendimento que você tinha sobre Alzheimer mudou?

Após a coleta, transcrição e organização dos dados, foi utilizado para a análise o Discurso do Sujeito Coletivo (DSC), que é um método que propõe a soma de ideias, de forma não numérica, que operacionalizadas metodologicamente expressem o pensamento de um determinado grupo por meio do discurso. Entende-se o DSC como um projeto de organização e tabulação de informações qualitativas de cunho verbal, obtidas de depoimentos que, 
basicamente, analisa o material verbal coletado para se extrair dele as Ideias-Centrais (IC) e suas correspondentes ExpressõesChaves $(\mathrm{ECH})^{(10)}$. Esse material verbal coletado na primeira pessoa do singular se transforma em uma referência coletiva, pois ocorre a associação discursiva que reúne fragmentos de distintas falas em um único discurso.

A investigação foi iniciada após a aprovação do projeto pelo Comitê de Ética em Pesquisa (CEP) da Universidade Federal de Campina Grande (UFCG), campus de Cajazeiras, sob o número do parecer 2.904.843 em 19 de setembro de 2018. A participação na pesquisa se realizou mediante a assinatura do entrevistado no Termo de Consentimento Livre e Esclarecido (TCLE), elaborado em duas vias, assinado pelo participante da entrevista e pela pesquisadora responsável. Respeitando a resolução 510/2016 do Ministério da Saúde, os componentes éticos e legais estão presentes em todas as etapas da pesquisa, assegurando aos participantes o sigilo e privacidade das informações que foram coletadas.

Os entrevistados foram identificados com codinomes de fenômenos naturais: céu; nuvem; dia; estrela; noite; terra; árvore e mar.

\section{RESULTADOS}

Para a execução das ações educativas, foram elaborados temas após o diagnóstico situacional da pesquisa-ação juntos aos participantes da pesquisa, estes foram: Conhecendo a Doença de Alzheimer e trabalhando os sentimentos de altruísmo e Dividindo experiências acerca da doença e reafirmando o ato da espiritualidade como força para superar os obstáculos.

A primeira ação ocorreu no dia 31 de outubro de 2018, em que, foram trabalhadas duas temáticas: o vivenciar no cuidado a pessoa idosa com Alzheimer como possibilidade de aprendizagem sobre a doença e o sentimento de altruísmo no cuidado a pessoa idosa com Alzheimer. Neste primeiro encontro estiveram presentes sete cuidadores.

Ao chegarem, os participantes foram acolhidos e orientados a sentar em círculo. Em seguida, realizou-se a apresentação expondo os objetivos da pesquisa e esclarecendo sobre o estudo e a temática a ser discutida naquela noite.

A aproximação dos participantes facilita na condução das atividades, dessa forma, foi realizada a dinâmica de acolhimento do "Guiar e ser guiado" com finalidade de demostrar a importância de saber conduzir e superar os obstáculos que possam ocorrer durante o período da doença. Para realização foi solicitado que formassem duplas sendo que cada um realizaria a 
condução do outro que estava vendado por obstáculos dispostos pelo salão de forma que preservasse a integridade do que estava sendo guiado, construindo um momento de descontração e harmonia do grupo.

A primeira metodologia utilizada foi a da "Árvore do conhecimento" com a finalidade de debater sobre conceito, sinais e sintomas, diagnóstico e tratamento da Doença de Alzheimer, desta forma, um ouvindo a fala do outro compartilha saberes.

Com a sala disposta em roda de conversa, o desempenhar da atividade se deu pela impressão de imagens que retratassem os princípios e características principais do Alzheimer como a história da doença, a perca da memória, a agressividade, o sentimento de angustia, os tratamentos a não aceitação dos cuidados entre outros, que foram entregues aleatoriamente, porém seguiam uma sequência lógica de apresentação, e cada participante iria expor e retratar o significado da imagem para os outros participantes. Em seguida, a facilitadora explicava com base científica dos artigos e documentos ministeriais o que cada imagem representava, após elas eram expostas em um mural desenhado no formato do cérebro para ocorrer a fixação visual.

A segunda metodologia consistiu em abordar e estimular o compartilhamento dos sentimentos gerados pela doença de forma que os estimulassem a desenvolver ainda mais o sentimento altruísta demostrado nas entrevistas. Para isso foi utilizada a metodologia do "Ateliê", em que eles iriam construir por meio do seu conhecimento. Previamente foi solicitado que todos trouxessem de casa uma ou mais fotos reveladas do idoso sob sua responsabilidade.

A facilitadora dispôs de folhas brancas, cola, pincéis, lápis coloridos, canetas, tesoura, adesivos, EVA's com recortes em vários formatos, imagens do tipo emoticons, gliter e imãs sobre a mesa e solicitou que os cuidadores colassem as fotos e em seguida com a utilização do material ofertado demostrassem o sentimento que eles possuíam, bem como sua relação com o cuidado, sendo acompanhados ao fundo de músicas clássicas aleatórias para propiciar ambiente agradável. Posteriormente foi solicitado que todos expusessem seu produto final e falassem sobre sua imagem e o que ela representa.

Com essa atividade pode-se notar que cada um se concentrou bastante e se engajou na atividade, tratando o momento com leveza e tranquilidade. Em meio ao material existiam representações que poderiam demostrar sinais de insatisfação, tristeza, sofrimento ou algo do gênero, porém, no momento da exposição todos demostraram sentimentos positivos principalmente de amor, com representação 
de um coração em todas as produções, como também por meio das falas das "obras de arte" criadas. Muitos utilizaram de sua imaginação para criar um belo painel, que foi sugerido pelos cuidadores deixar exposto em suas residências.

O segundo encontro ocorreu no dia oito de novembro de 2018, e teve como temáticas a espiritualidade como ferramenta do cuidado a pessoa idosa com Alzheimer e ausência de suporte na realização dos cuidados cotidianos a pessoa idosa com Alzheimer. A ação consistiu em compartilhar e buscar soluções para as dificuldades diárias na realização do cuidado com base na metodologia da "Situação-Problema", além de concluir o encontro reafirmando a busca pela espiritualidade como ferramenta de ânimo e fé para superar as dificuldades. Participaram desta etapa oito cuidadores.

A primeira metodologia ativa estava voltada para a sobrecarga do cuidador. Previamente foram colocados em balões papeis com situações do cotidiano que eles citaram durante a entrevista que diagnosticava a sobrecarga, sendo mantido o anonimato do entrevistado. Os balões foram entregues aleatoriamente e eles foram orientados que se fosse sua fala não era necessário revelar. $\mathrm{Na}$ sequência a pesquisadora solicitou que formassem duplas e que um iria ler a situação-problema e o outro iria buscar uma forma de resolvê-la, em seguida os demais iriam dizer se concordam com a solução proposta e se dariam novas alternativas para resolubilidade do problema.

Os participantes demostraram interesse em participar através da fala, podendo se notar que ao longo da metodologia com a exposição das situaçõesproblema, eles se identificavam com a fala do outro como algo que eles também vivenciam e alguns expuseram a fala como sendo a sua. Foi de suma importância esse momento, pois através da conversa eles buscaram solucionar o problema do outro e se viram em situações que eles ainda não tiveram a experiência, mas que poderiam ainda vivenciar.

O segundo momento consistiu em duas etapas de metodologias sendo a primeira o uso do jogo "quebra-cabeça" que formava uma imagem que se relacionava a espiritualidade. Os cuidadores foram orientados a seguir para a mesa em que estava disposto o jogo. Em coletividade todos deveriam montar o quebra-cabeça, para que assim, seguissem para a próxima etapa.

Durante a montagem do quebracabeça pode-se observar falta de otimismo em conseguir solucionar o jogo, isso também reflete nos cuidados diários, principalmente com pessoas idosas com DA que devido as fases da doença tem que se juntar peças de um "quebra-cabeça" que não há um modelo e 
assim conseguir desempenhar as atividades diárias e não apenas buscar o caminho mais fácil ou se entregar diante das dificuldades. Porém, em outra perspectiva, alguns participantes mostraram-se com força de vontade e empenho para montar o jogo, sempre estimulando os demais participantes e traçando estratégia para que concluíssem a atividade.

A segunda etapa consistiu no momento de reflexão acerca de como os cuidadores exercitam sua espiritualidade. Foi previamente organizado espaço agradável em que os participantes se sentariam sobre um tapete ao chão, acompanhados de comidas, músicas de relaxamento e ambiente a meia luz. Nesse espaço a pesquisadora levantou a questão se eles sabiam a diferença entre religião e espiritualidade, sendo que em unanimidade não souberam diferenciar, desta forma, a facilitadora explicou a diferença. Logo após eles voluntariamente externaram seus sentimentos espirituais acerca das condições vividas por eles e pelos idosos que cuidam.

Nesse momento também foi utilizado o "Jogo da Garrafa" para que cada um estimulasse o outro com apenas uma palavra que os motivasse a seguir em frente, e por meio do jogo pode-se notar que as palavras fé e paciência foram as mais recorrentes.
Após as intervenções, objetivou-se analisar se as ações repercutiram no entendimento do cuidador em relação às temáticas abordadas. Desta maneira, foram realizadas entrevistas com os cuidadores que participaram das ações, o que formou a IC de discussão denominada: Compartilhando experiências como forma de aprendizagem na transformação dos cuidados ofertados.

A IC aborda a percepção dos cuidadores diante da experiência de participar de ações educativas e a agregação do conhecimento advindo das ações. Para a construção do Discurso do Sujeito Coletivo (DSC) dessa categoria, participaram oito cuidadores (céu, nuvem, dia, estrela, noite, terra, árvore e mar).

\section{IC- Compartilhando experiências como forma de aprendizagem na transformação dos cuidados ofertados.}

DSC: É uma tarefa muito difícil viver em função de outra pessoa, cansa muito, tem hora que você precisa de um apoio, precisa de outras experiências, até para melhorar a assistência que você da à pessoa que tá com Azheimer e gostei bastante pincipalmente da forma como você abordou, as dinâmicas... você vê que o problema não é só na sua família, você vê que tem outras pessoas, que apesar de não ter cura, mas pelo menos você acalenta um pouco a preocupação que você tem, a tristeza que você passa... a gente adquire muita experiência um com o outro, compartilhar conhecimentos e aprender um pouco mais sobre a doença. Eu tenho certeza que se eu não tivesse participado eu não teria a visão que eu 
tenho agora no momento, que eu sabia que a doença em si ela já trazia consequências, mas eu vi de uma forma diferente e dinâmica que deu pra mim aprimorar realmente tudo que é a doença.

\section{DISCUSSÃO}

A IC proporciona a visão de que para mudar a realidade do conhecimento deficiente é necessário que se dinamize o modo como às informações são compartilhadas, constatando que a aprendizagem baseada na troca de experiências acalenta os problemas vivenciados no cotidiano, pois são comuns para todos os cuidadores envolvidos no processo da DA.

A pesquisa-ação consegue proporcionar vínculo entre o pesquisador e os participantes, fazendo com que haja reflexão para solucionar os problemas ${ }^{(11)}$. Tornando os cuidadores sujeitos ativos no processo de troca de saberes, e os conduzindo no planejamento e construção do saber.

Observa-se no discurso dos cuidadores, que participar das ações em saúde proporcionou mudanças na concepção que tinham sobre a DA, e consequentemente melhorias tanto para o cuidador que aprofundou seu conhecimento, quanto para o idoso que terá suas necessidades supridas com um olhar diferenciado de seu cuidador.

Cuidar de um familiar com DA requer que o cuidador saiba conviver com o sofrimento do outro. Dessa forma, é necessário desenvolver ações que proporcionem diálogos, respeitando os saberes e crenças e promovam capacitação aos cuidadores, pois o cuidador bem informado se torna mais seguro, refletindo em maior capacidade e disponibilidade para os cuidados ofertados ${ }^{(12)}$.

O estudo realizado com cuidadores familiares participantes dos grupos da Universidade de São Paulo demonstrou em seus achados, que as cuidadoras se interessaram na busca e acesso aos grupos de apoio psicoeducacional da Associação Brasileira de Alzheimer como possibilidade de troca de experiências e orientação no manejo dos sintomas da $\mathrm{DA}^{(13)}$.

Outro estudo realizado no Rio Grande do Sul com familiares cuidadores de pessoas idosas com DA, demonstrou que os participantes perceberam que com o grupo conseguiram aprender sobre a DA e sobre como cuidar da pessoa idosa e ao mesmo tempo, sentiam-se cuidados, uma vez que o grupo proporcionou troca dialógica onde o cuidador conseguia aprender e ensinar, melhorando suas práticas de cuidado ${ }^{(14)}$.

A criação de grupos facilita a interação entre pessoas que partilham da mesma experiência, como é o caso dos cuidadores de pessoas idosas com DA. Isso porque um cuidador que participa de 
intervenções em habilidades sociais com profissionais de saúde e outros cuidadores, se sente seguro em indagar sobre aspectos da doença, além de transparecer seus sentimentos e dialogar quando algo está lhe incomodando ${ }^{(15)}$.

Dessa forma, o processo de educação consiste também na perspectiva de mudanças do cuidador, almejando melhorias de um cuidado qualificado com a pessoa idosa dependente que vive em domicílio ${ }^{(16)}$.

Com isso, é necessário que se fortaleçam os diálogos baseados nas experiências, possibilitando o uso da realidade para subsidiar discussões e efetivar o aprendizado, que com metodologias ativas se torna mais estimulante e incentivador ${ }^{(17)}$.

Por fim, como diagnosticado na fala dos cuidadores, as metodologias educativas contribuem para que haja engajamento dos participantes e para que o conhecimento seja descomplicado, mudando a perspectiva acerca do cuidado de pessoas idosas com DA proporcionando conforto, segurança e empoderamento.

\section{CONSIDERAÇÕES FINAIS}

O presente estudo proporcionou a análise e discussão da educação em saúde com cuidadores informais de idosos com doença de Alzheimer.
Na realização da análise das ações com os cuidadores foi perceptível que as metodologias ativas utilizadas proporcionaram espaço de troca de conhecimentos, pois a interação em grupos educativos permitiu que cuidador percebesse que os problemas que o assolam, são semelhantes com o que outros cuidadores vivenciam.

Além disso, o compartilhamento de saberes modificou a forma do cuidador em perceber o que é a DA, favorecendo a assistência ofertada ao idoso.

Cabe salientar que os resultados obtidos nesta investigação apresentam limitações, já que com o levantamento dos dados, foi obtido um número maior de cuidadores no município, porém alguns não quiseram participar das ações. Além disso, os achados fazem parte da realidade de um munício específico o que dificulta grandes generalizações já que trata de uma realidade local.

Por fim, é necessário promover a educação aos cuidadores de pessoas idosas com DA, buscando sanar as principais dificuldades e utilização de novas tecnologias em saúde. Acredita-se que por meio da educação em saúde haverá a diminuição dos agravos na saúde da pessoa idosa com DA e do cuidador, que se dedica fielmente ao cumprimento de suas atividades. 


\section{REFERÊNCIAS}

1. Silva SPZ, Bernardo AV, Lô CLN, Campeiro GVT, Santos LR. Assistência de enfermagem aos pacientes portadores de alzheimer: uma revisão integrativa. Revista Nursing. 2020. 23(271): 4991-4. Disponível em:

https://doi.org/10.36489/nursing.2020v23i271 p4991-4998

2. Moreira ML, Bucher-Maluschke JSNF, Silva JC, Falcão DVS. Cuidadores informais de familiares com Alzheimer: vivências e significados em homens. Contextos Clínicos. 2018. 11(3): 373-85. Disponível em: https://doi.org/10.4013/ctc.2018.113.08

3. Alzheimer's association. Alzheimer's disease facts and figures. Alzheimer's Dement 2020.16: 391-460. Disponível em: https://doi.org/10.1002/alz.12068

4._Pocinho R, Belo P, Melo C, NavarroPardo E, Muñoz, JJF. Relação entre o estado psicossocial do cuidador informal e o tempo de cuidado dos idosos da região centro de Portugal. Educación Y Humanismo. 2017. 19(32), 88-101. Disponível em: https://doi.org/10.17081/eduhum.19.32.2533

5. Rosas-Cervantes E, Valenzuela-Suazo S, Jiménez-González

MJ. Spiritual transcendence, opportunity of informal careers of the elderly: integrative review from the Watson philosophy. Enfermería Montev. 2020. 9 (2): 149-59. Disponível em: http://www.scielo.edu.uy/scielo.php?script=sc i_arttext\&pid=S2393-

66062020000200149\&lng=es\&nrm=iso\&tlng $=$ en

6. Garbaccio J, Tonaco LAB. Characteristics and Difficulties of Informal Caregivers in Assisting Elderly People. J. res.: fundam. care. online. 2020. 11(3): 680-6. Disponível em: $\quad$ https://dx.doi.org/10.9789/2175$\underline{\text { 5361.2019.v11i3.680-686 }}$
7. Perdigão LMNB, Almeida SC, Assis MG. Estratégias utilizadas por cuidadores informais frente aos sintomas neuropsiquiátricos de idosos com demência. Rev. Ter. Ocup. Univ. São Paulo (Online). 2017. 28(2):156-62. Disponível em: https://doi.org/10.11606/issn.22386149.v28i2p156-162

8. Thiollent M. Metodologia da pesquisaação. 18. ed. São Paulo: Cortez, 2011.

9. Instituto Brasileiro de Geografia e Estatística (IBGE). Cidades e Estados. Disponível em:

https://www.ibge.gov.br/cidades-eestados/pb/sao-jose-de-piranhas.html

10. Lefèvre F, Lefèvre AMC. O discurso do sujeito coletivo: um novo enfoque em pesquisa qualitativa (desdobramentos). Caxias do Sul: Educs; 2005.

11. Dias ESM, Rodrigues ILA, Miranda HR, Corrêa JA. Roda de conversa como estratégia de educação em saúde para a enfermagem. J. res.: fundam. care. online. 2018. 10(2): 379$84 . \quad$ Disponível em: https://dx.doi.org/10.9789/21755361.2018.v10i2.379-384

12. Kucmanski LS, Zenevicz L, Geremia DS, Madureira VSF, Silva TG, Souza SS. Alzheimer's desease: challenges faced by family caregivers. Rev. bras. geriatr. gerontol. 2016. 19(6): 1022-29. Disponível em: https://doi.org/10.1590/198122562016019.150162

13. Mattos EBT, Kovács MJ. Doença de Alzheimer: a experiência única de cuidadores familiares. Psicol. USP. 2020. 31(e180023):111. Disponível em: https://doi.org/10.1590/0103-6564e180023

14. Ilha S, Santos SSC, Backes DS, Barros EJL, Pelzer MT, Costenaro RGS. Complex educational and care (geron)technology for 
elderly individuals/families experiencing Alzheimer's disease. Rev. Bras. Enferm. 2017. 70(4): 726-32. Disponível em: https://doi.org/10.1590/0034-7167-2016$\underline{0687}$

15. Queluz FNFR, Barham EJ, Prette ZAPD, Santos AAA. Social Skills Inventory for Caregivers of Elderly Family Members (SSI$\mathrm{CE})$ : Relations with Indicators of Psycholgical Wellbeing. Trends in Psychology. 2018. 26(2), 537-564. Disponível em: https://doi.org/10.9788/tp2018.2-01pt

16. Lima AAC, Carvalho FLQ, Lima JRO. O cuidador da pessoa idosa: processo educativo ou formação para o mercado? Revista Interdisciplinar em Educação e Territorialidade - RIET. 2020. 1(1): 245-57. Disponível em: https://doi.org/10.30612/riet.v1i1.12656

17. Silva RP, Camacho ACLF, Silva MAP, Menezes HF. Strategies for the use of active methodology in the training of nursing academics: experience report. Research, Society and Development. 2020. 9( 6): e160963543. Disponível em: https://doi.org/10.33448/rsd-v9i6.3543

\section{Autor correspondente} Daniele Pereira Soares. Endereço: Rua Francisco Beltrão, 90, apto 501. Jardim Oceania, João Pessoa, Paraíba, Brasil, CEP: 58.037-605. Telefone: (83) 99127-8694. Email: danisoaresenf@gmail.com.

Submissão: 2021-06-13

Aprovado: 2021-07-13 